\title{
Comparison of Erbium, Raman and Parametric Optical Fiber Amplifiers for Burst Traffic in Extended PON
}

\author{
Chandra B Gaur, Filipe Ferreira, Vladimir Gordeinko, Asif Iqbal, Wladek Forysiak and Nick Doran \\ Aston Institute of Photonic Technologies, Aston University, Birmingham, U.K \\ gaurc@aston.ac.ukl
}

\begin{abstract}
Experimental comparison of burst traffic amplification by polarization independent fiber optic parametric amplifier, discrete Raman fiber amplifier and an erbium doped fiber amplifier. Parametric amplification improves required received power by more than $3 \mathrm{dBs}$.

(C) 2020 The Author(s) OCIS codes: (060.2330) Fiber optics communications; (060.2320) Fiber optics amplifiers and oscillators.
\end{abstract}

\section{Introduction}

Current physical reach in 10G-PON passive optical networks is limited to $20 \mathrm{kms}$ due to chromatic dispersion and power budget allowance in direct detection systems [1].Reach extension in PON is required to extend number of users and also to consolidate physical metro-access network layers hence to reduce multiple active nodes [2].Future access networks will evolve current state-of-the-art PON to enhanced dispersion tolerable transceiver solutions using coherent and advanced digital signal processing. But to increase power budget allowance in-line amplification is required parallelly enhancing point to multipoint capacity at branching nodes. However, amplifiers should accommodate compatibility with burst transmission of upstream PON signal due to time division multiplexing. As readily available erbium doped fiber amplifiers (EDFAs) may have problems with the inevitable bursty nature of the traffic due to metastable lifetime of erbium ions ( $1 \mathrm{~ms})$ [3]. Hence other two potential candidates are parametric amplifiers and Raman amplifiers. Recent development in discrete Raman fiber amplifiers (DRFAs) for optical communication networks have recognized its potential as an in-line amplifier [4]. With almost instantaneous response time ( $\sim 76 \mathrm{fs})$ stimulated Raman scattering (SRS) effect for Raman amplifiers is very fast [5]. Saturation in DRFAs due to net gain requirement comes from high pump powers [6]. However, for burst traffic transmission with counter propagating pumps DRFAs transient response time becomes of the same order of magnitude of the propagation delay along the gain fiber.[6]. (e.g. $5 \mu \mathrm{sec}$ for each gain fiber km). Moreover, the gain transient is further enhanced by the saturated nature of practical DRFAs [6]

Fiber Optical Parametric Amplifier (FOPA) shows almost instantaneous response time $(0.1 \mathrm{fs})$ scale as related to Kerr effect response time, presents FOPA as a potential candidate for access networks as in-line amplifier [7]. Recent work in [8] overcome their inherent polarization dependent gain nature using a loop architecture to deliver a polarization independent FOPA. Compatibility of FOPA to work in arbitrary wavelengths also make them interesting for O-band amplification [9]. They are already of potential research interest due exceptional features like high gain with large and flat bandwidth [10]. In this paper we experimentally demonstrate amplification of different duration 10Gbps burst signal with PI- FOPA compare with a commercial grade EDFA and for the first time with a DRFA, this way extending our PI-FOPA versus EDFA results.in [11] Our results show a significant difference in performance of the FOPA from the other two amplifiers. Specifically, we see a $3 \mathrm{~dB}$ better performance for the FOPA in power budget than DRFA for short $10 \mu \mathrm{sec}$ burst duration and an even larger difference for the EDFA. These results indicate a new area of application for reach extended access network as an in-line amplifier.

\section{Experimental Setup}

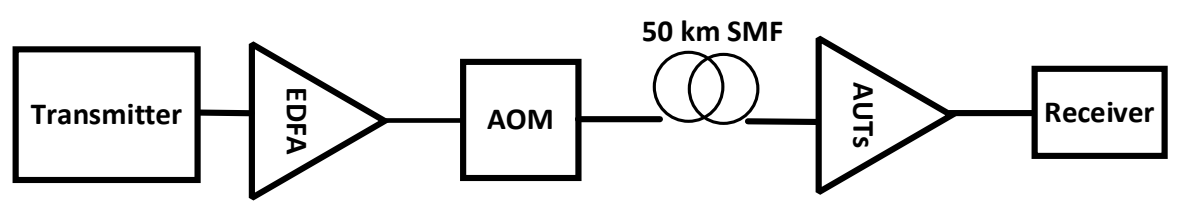

Fig.1: Schematic of experimental setup of burst mode amplifier

Figure 1 shows experimental setup used in this demonstration also explained detail in [11]. We have 10Gbps nonreturn-zero (NRZ) modulated signal generated using an MZM and tunable laser source at $1535 \mathrm{~nm}$. Modulated signal amplified by booster EDFA and the burst is created by using Acousto Optic Modulator (AOM) with fixed 
$10 \mathrm{kHz}$ square pulse generated from waveform generator to emulate the bursty nature of real traffic to provide constant burst periods. Burst with different durations are then transmitted over $50 \mathrm{~km} \mathrm{SMF}$, propagated signal is amplified via amplifiers under test (AUTs). Variable optical attenuator (VOA) is used to mimic as 1:16 optical splitter (split count limited by DRFA 13dB saturated gain). Receiver consist of band pass filter (BPF) of $0.3 \mathrm{~nm}$ bandwidth, a direct detection receiver and real-time oscilloscope to analyze signals. Experimental setup of discrete Raman fiber amplifier (DRFA) used for bursts amplification is as follows. High power Raman pump at $1452 \mathrm{~nm}$ is pumped in backward direction of $6.5 \mathrm{~km}$ of dispersion shifted fiber (DSF). Pump power of $2 \mathrm{~W}$ is used to amplify signal propagating in forward direction. DRFA operates in saturation at $13 \mathrm{~dB}$ net output gain to amplify $-2 \mathrm{dBm}$ input signal power. Zero dispersion wavelength of DSF was at $1542 \mathrm{~nm}$. High power isolators were used to protect any back reflections in the pump source. WDM coupler was used to remove pump after amplification. Output amplified signal was then transmitted to receiver via VOA. PI-FOPA used operates in a. loss-gain configuration [8], it works as follows. Input signal from transmitter and after $50 \mathrm{~km}$ SMF, is splitted by polarization beam splitter (PBS) in two separate polarization states. Each polarization state of signal is amplified by 200 and 250 mtrs of highly non-linear fiber (HNLF) by separate gain arms, different pumps are inserted into each arm by WDM couplers in feed-in feed-out arrangement. Pump power in each arm was $35.4 \mathrm{dBm}$ and $34.4 \mathrm{dBm}$ to balance pump power and gain. Net gain of $13 \mathrm{~dB}$ was achieved in polarization insensitive state via recombining $\mathrm{X}$ and $\mathrm{Y}$ polarization states of amplified signal at the output of the setup. Also working principle is also explained in detail in $[8,11]$.

\section{Results and Discussion}

In this section we will analyze bit error ratio (BER) performance evaluation for non-burst to burst traffic amplification by different AUTs. First as a baseline comparison non-burst amplification was performed for 10Gbit/s signal with $13 \mathrm{~dB}$ gain. From Fig. 2(a) it is observed that around $1 \mathrm{~dB}$ received power penalty was noticed between $\mathrm{B} 2 \mathrm{~B}$ and different amplifiers in non-burst mode. It can be understood from the baseline figure that in non-burst mode relative performance for different amplifiers demonstrate similar effect with BER measurements. Also, to note low gain amplification (13dB) in EDFA creates higher noise figure which degrades its performance. From previous work in [11], we can comment that with amplifier gain of $20 \mathrm{dBs}$ in non-burst mode EDFA performance surpassed that of the FOPA for the range of signal power tested.

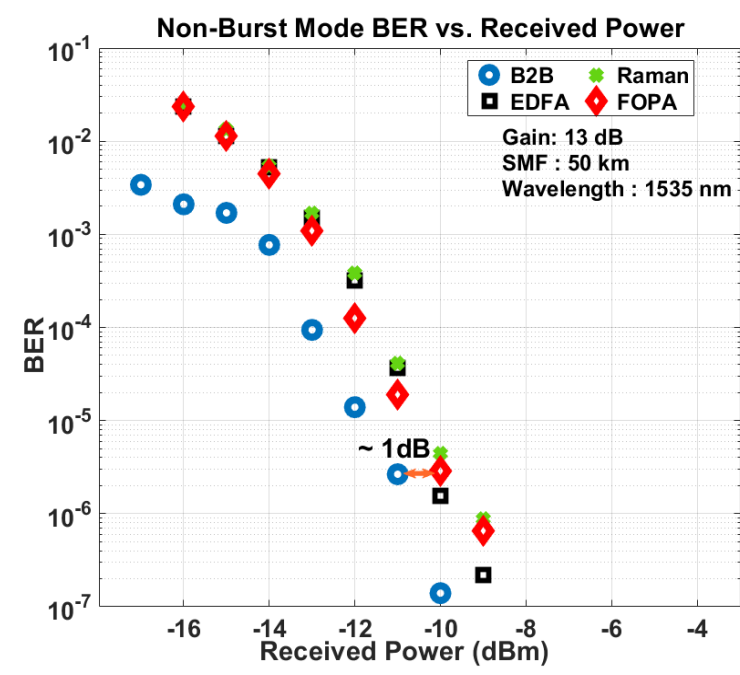

Fig 2(a): BER vs Rx. Power Curve for non-burst mode signal

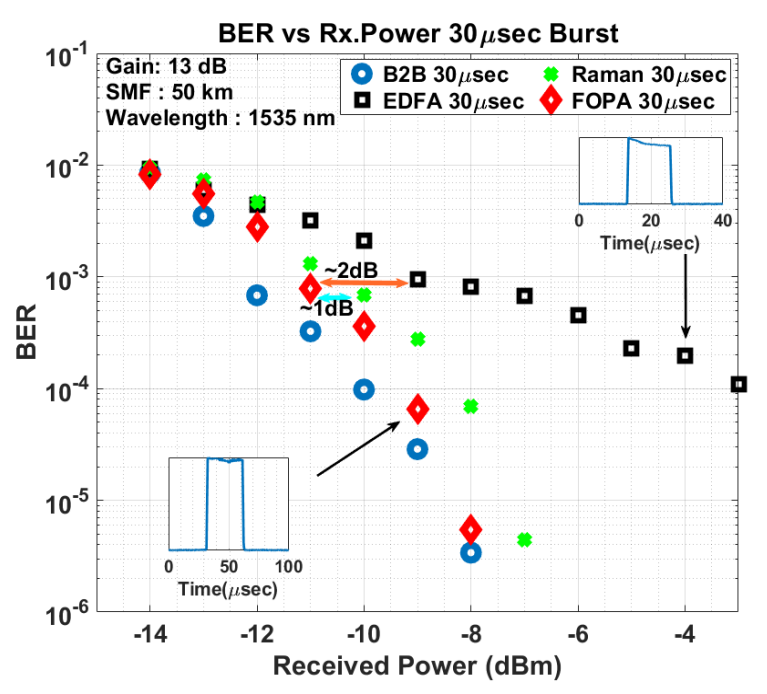

Fig 2(b): BER vs Rx. Power plot for 30 $\mu$ sec Burst Mode Signal

Figure.2(b) shows BER performance for $30 \mu \mathrm{sec}$ burst duration signal amplification. Overshoots at the leading edge of EDFA amplified bursts are noticed as shown in the right figure inset, whereas no such effect was observed in FOPA amplified burst shown in bottom left inset. Similarly, no significant performance degradation in comparison with FOPA was noticed in DRFA amplified 30 $\mu$ sec burst signal. Appearance of noise floor is visible in EDFA due to leading edge of burst faces high gain fluctuations and long relaxation time to reach steady state observed due to high gain fluctuations which corrupts multiple number of bits at leading edge of the burst. Fast response time in FOPA protects bits from degradation due no apparent gain fluctuation. In Fig 3(a). BER performance of 10 $\mu$ sec burst duration amplification is experimentally investigated by all different AUTs. Severe degradation in BER was 
observed for both DRFA and EDFA amplified bursts. High overshoots in EDFA creates non recoverable bursts, while DRFA amplified burst shows large received power penalty between FOPA and DRFA, which is observed around $\sim 3 \mathrm{~dB}$. Although it is observed that for $10 \mu \mathrm{sec}$ burst no noticeable BER degradation was found in FOPA. It can be commented that due to high incurring transient fluctuations is visible for both DRFA and EDFA amplifiers incur degradation in burst amplification.

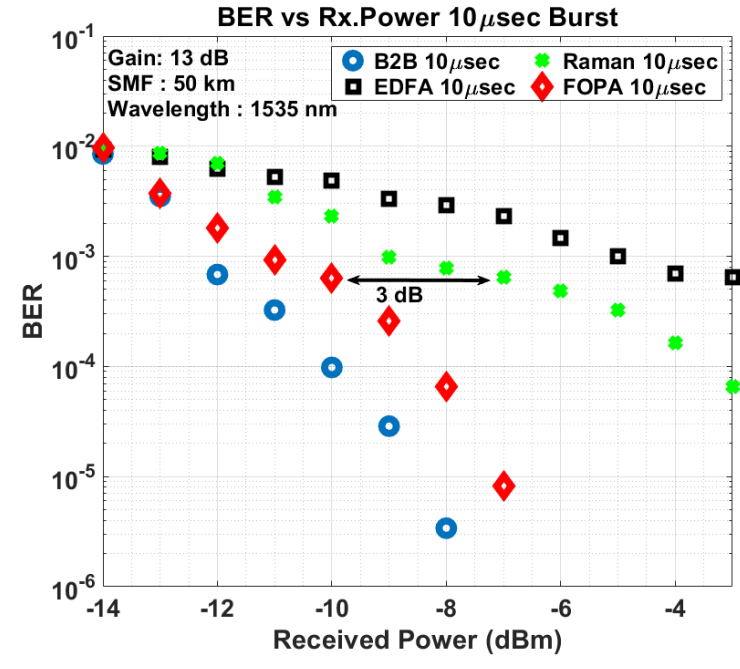

Fig.3: BER vs Rx. Power curve of $10 \mu \mathrm{sec}$ Burst Signal

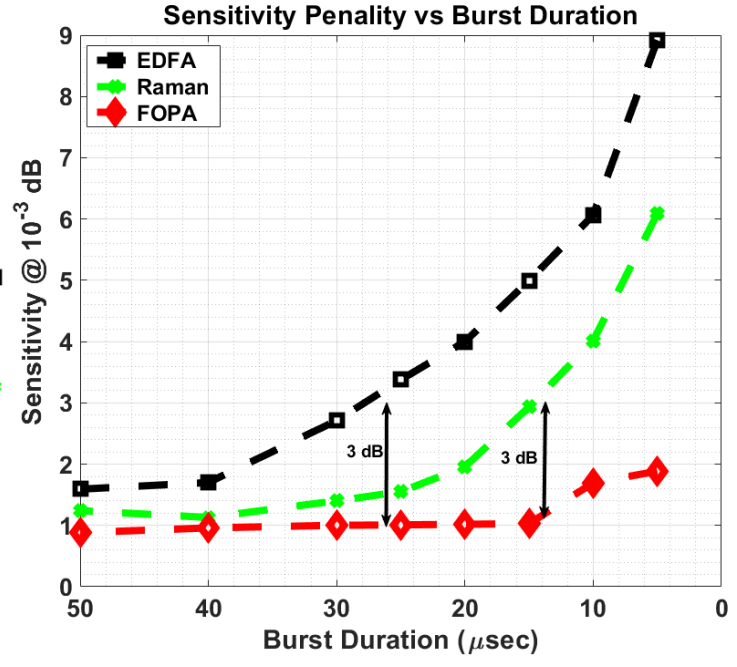

Fig .4: Sensitivity Penalty vs Burst Duration

Figure 4 above shows required power penalty for $\mathrm{BER}=10^{-3}$, taking as reference the $\mathrm{B} 2 \mathrm{~B}$ required power, as a function of burst duration. FOPA performance shows negligible dependency on the burst duration as expected given its response time. Therefore, in the following we refer to the additional penalty introduced by the EDFA and DRFA. The EDFA starts introducing a significant penalty for bursts shorter than $40 \mu \mathrm{s}$, with a penalty larger than $3 \mathrm{~dB}$ for bursts shorter than $20 \mu \mathrm{s}$. The DRFA performs better than the EDFA allowing amplification of shorts as low as $20 \mu \mathrm{s}$ without significant penalty. However, DRFAs introduce a penalty larger than $4 \mathrm{~dB}$ for bursts shorter than $5 \mu \mathrm{s}$, related to the propagation delay due to Raman gain medium.

\section{Conclusion}

We experimentally demonstrate and report amplification of dynamic duration 10Gbps burst signal amplification with PI-FOPA, DFRA and EDFA with $13 \mathrm{~dB}$ gain. FOPA provides around 3dB less penalty in received power

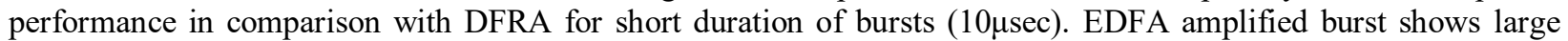
gain fluctuations which demonstrate degraded burst in comparison with FOPA below $40 \mu$ sec duration. This work shows that FOPA performance show no noticeable penalty due transient effects for multiple duration of burst amplification. Upstream burst signals in long reach PON may requires instantaneous gain compensation due to optical distribution network losses. In lieu with that FOPA can provide potential reach extension with large split ratio in long reach PON applications.

This work is supported by the EPSRC Grants: EP/M005283/1-UPON, EP/R024057/1-FOPA-ROCS. To access the underlying data please see research data. https://doi.org/10.17036/researchdata.aston.ac.uk.00000437

\section{References}

[1] ITU-T Recommendation ITU-T G.987.1 Long Reach: Gigabit-capable passive optical networks (GPON): General Characteristics

[2] D. B Payne et al, "End-to-end network design and experimentation in the DISCUS project.", ICTON, 2017, p Th. B1.2

[3] M Shiraiwa et al," Performance evaluation of a burst-mode EDFA in an optical packet and circuit integrated network", Opt. Expr ,21,2013

[4] M. Dalla Santa et al, "Burst-mode analysis of XGPON Raman reach extender employing quantum-dot lasers." Electr Lett 52,1157-58, 2016

[5] R.H Stolen et al, "Raman response function of silica-core fibers.", JOSA B,6, 1159-1166. (1989)

[6] C J Chen et al, "Transient effects in saturated Raman amplifiers." Electr Lett ,37, 6, 2001

[7] M.S-Bahae and M Hasselbeck," Third Order Non-linearity, optical Kerr effect “, (OSA) Handbook of Optics, Chap.4

[8] V Gordienko et al, "Suppression of Nonlinear Crosstalk in a Polarization Insensitive FOPA by Mid-stage Idler Removal", OFC 2019, p. M4C.-4.,2019

[9] Xing Xu, et al "Distributed parametric amplification at $1.3 \mu \mathrm{m}$ in 25-km single-mode fiber." Int. Conf. on PS, pp. 1-3. IEEE, 2012.

[10] V. Gordienko et al," Demonstration of an ultra-flat Raman-enhanced fibre optical parametric amplifier (FOPA) with $>110 \mathrm{~nm}$ gainbandwidth", ECOC 2016

[11] C B Gaur et al, "Demonstration of improved performance by FOPA for extended PON in burst-mode operation", in ECOC 2019 p.Th.1. C.4, 